\title{
HIF-1 $\alpha$ may provide only short-term protection against ischemia-reperfusion injury in Sprague-Dawley myocardial cultures
}

\author{
SIYANG WANG ${ }^{*}$, XIN SHAO* ${ }^{*}$ XIAOXUE LI, XIAOJUAN SU, YONGXU HUO and CHUNLEI YANG \\ Department of Life Sciences, Sichuan University, Chengdu, Sichuan 610041, P.R. China
}

Received October 21, 2015; Accepted January 22, 2016

DOI: $10.3892 / \operatorname{mco} .2016 .764$

\begin{abstract}
Hypoxia-inducible factor-1 (HIF-1 $\alpha$ ) exerts an important role in protecting against cardiac tissue damage, for example, following ischemia-reperfusion (I/R), although the time frame during which it acts has yet to be fully elucidated. In the present study, a culture model of myocardial cells from Sprague-Dawley rats was used to examine the expression levels of HIF-1 $\alpha$ and various downstream effectors at different times following I/R. The levels of HIF-1 $\alpha$ were manipulated by overexpressing HIF-1 $\alpha$ prior to I/R. HIF-1 $\alpha$ levels peaked at $6 \mathrm{~h}$ following I/R, subsequently decreasing to low levels. The levels of downstream effectors peaked at $48 \mathrm{~h}$, and decreased almost to pre-I/R levels by $72 \mathrm{~h}$. These results suggest that HIF-1 $\alpha$ and its downstream targets offer only short-term protection following I/R. These results may have implications for the treatment of I/R-associated injury in a variety of clinical contexts.
\end{abstract}

\section{Introduction}

Although the restoration of blood flow to ischemic organs is essential to prevent irreversible tissue damage, reperfusion is able to trigger local and systemic inflammation, which leads to more severe tissue injury than ischemia alone (1). Such ischemia-reperfusion (I/R) injury may occur in a variety of clinical contexts, including thrombolytic therapy, coronary angioplasty, organ transplantation, aortic cross-clamping and cardiopulmonary bypass (1). Several approaches have been applied in the clinic to treat I/R injury (2), with perhaps the best-studied example being ischemic preconditioning (3). This involves inducing discontinuous ischemic episodes prior to the sustained ischemia.

Correspondence to: Professor Chunlei Yang, Department of Life Sciences, Sichuan University, III Middle Segment, 17 Renmin South Road, Chengdu, Sichuan 610041, P.R. China

E-mail: yangchunlei@scu.edu.cn

*Contributed equally

Key words: HIF-1 $\alpha$, ischemia/reperfusion, myocardial cells
Hypoxia-inducible factor-1 (HIF-1), comprising HIF-1 $\alpha$ and HIF-1 $\beta$ subunits (4), is a central regulator of oxygen homeostasis. HIF-1 $\alpha$ is universally expressed in vertebrates, insects, worms and other species (5). Under conditions of normoxia (normal oxygen), the ubiquitin-proteasome pathway maintains levels of HIF-1 $\alpha$ at a minimum (6); in hypoxia, however, HIF-1 $\alpha$ is protected from proteolysis (1), leading to a rapid increase in protein levels. HIF-1 $\beta$, by contrast, is expressed constitutively at similar levels under normal and hypoxic conditions (7).

HIF- $1 \alpha$ activates the transcription of genes encoding proteins that mediate adaptive responses to hypoxia/ischemia, including erythropoietin, glucose transporter 1, vascular endothelial growth factor (VEGF), as well as genes regulating cell survival (1). HIF-1 $\alpha$ may also help drive angiogenesis following tissue injury (8). The length of time during which HIF-1 $\alpha$ continues to influence the expression of downstream effectors following I/R has yet to be fully elucidated, which is important for understanding how HIF-1 $\alpha$ mitigates I/R injury. In the present study, the time-scale of HIF-1 $\alpha$ action was investigated using a culture model of myocardial cells derived from Sprague-Dawley rats.

\section{Materials and methods}

Animals. The study protocol was approved by the Animal and Ethics Review Committee at the Life Science Institute of Sichuan University in Chengdu, China. A total of 30 male Sprague-Dawley rats were purchased from the Animal Experiment Center of Sichuan University (animal license SCXK2006-010). The rats were kept under light conditions, and they were fed on normal rat fodder provided by the Animal Experiment Center of Sichuan University.

Construction of a eukaryotic expression plasmid encoding rat myocardial HIF-1 $\alpha$. Pspt18, a plasmid expressing full-length rat HIF-1 $\alpha$, empty expression vector pcDNA3.1 (Life Technologies; Thermo Fisher Scientific, Waltham, MA, USA) and Escherichia coli JM109 were kindly given by Professor Yi Qu of West China Second University Hospital (Chengdu, China). The HIF-1 $\alpha$-coding region was subcloned into pcDNA3.1 using BamHI (Takara Biotechnology Co., Ltd., Dalian, China) and DNA ligase Sol 1 (Takara Biotechnology Co., Ltd.), and the resulting expression plasmid was called 
$\mathrm{pHIF} \alpha$. Colonies apparently positive for inserts were screened by BamHI to confirm the presence of the insert.

Myocardial culture model of I/R injury. Myocardial cells were isolated from Sprague-Dawley rats, as previously described (2). Briefly, the pericardium and atrium were removed from 10 rat hearts in phosphate-buffered saline (PBS) buffer, and ventricular cells were separated using trypsin protease (Thermo Fisher Scientific). The cell suspension was passed through a $200 \mu \mathrm{m}$ screen into culture flasks. Cultures were maintained for 4 days under normal conditions $\left(37^{\circ} \mathrm{C}\right.$, $5 \% \mathrm{CO}_{2}$ ), prior to exposure for $30 \mathrm{~min}$ to hypoxic conditions $\left(37^{\circ} \mathrm{C}, 95 \% \mathrm{~N}_{2}, 5 \% \mathrm{CO}_{2}\right)$ to simulate ischemia. After $24 \mathrm{~h}$, the culture medium was replaced with Dulbecco's modified Eagle's medium (DMEM) containing 10\% fetal bovine serum (FBS) to simulate reperfusion (Sigma-Aldrich, St. Louis, MO, USA). In parallel, mock-I/R control cultures were maintained under normal conditions $\left(37^{\circ} \mathrm{C}, 5 \% \mathrm{CO}_{2}\right)$ for the same overall culture period. Cultures were harvested (by trypsin treatment and centrifugation at $1,000 \mathrm{rpm}$ for $5 \mathrm{~min}$ ) at $6 \mathrm{~h}$, or on day 5 following I/R.

Microscopy. Images were captured using a Nikon Eclipse Ti microscope (Nikon, Tokyo, Japan) at 100x magnification.

Transient transfections prior to $I / R$. Ventricular myocytes were prepared as described above, and cultured on 30-mm dishes until $80 \%$ confluence was reached. Subsequently, the medium was changed to DMEM without serum or antibiotics, and the cultures were transfected for $24 \mathrm{~h}$ with a mixture of $8 \mu \mathrm{g}$ pHIF $\alpha$ and $40 \mu$ l Lipofectamine ${ }^{\mathrm{TM}}$ (Invitrogen; Thermo Fisher Scientific). The cultures were subsequently grown in Dulbecco's modified Eagle's medium containing $10 \% \mathrm{FBS}$, and 100 units/ml penicillin and $100 \mu \mathrm{g} / \mathrm{ml}$ streptomycin (Sigma-Aldrich). In parallel, control cultures were mock-transfected with Lipofectamine ${ }^{\mathrm{TM}}$ alone. At 1 day following transfection, these transfected cultures were subjected to I/R or mock-I/R, as described above.

Measurement of myocardial cell viability using the methyl thiazolyl tetrazolium (MTT) assay. At $6 \mathrm{~h}$, or 5 days following $\mathrm{I} / \mathrm{R}$, cultures were harvested, resuspended in fresh medium and pelleted by centrifugation as previously described. The pellets were resuspended and inoculated in 96-well plates at a density of $5 \times 10^{3}$ cells per well. Plates were incubated for $24 \mathrm{~h}$, after which $20 \mu \mathrm{l}$ MTT reagent $(5 \mathrm{mg} / \mathrm{ml}$, Sigma-Aldrich) was added to each well. The plates were incubated for a further $4 \mathrm{~h}$, and subsequently the supernatant was removed. Dimethylsulfoxide (150 $\mu \mathrm{l}$; Sigma-Aldrich) was added to each well. The absorbance was measured at $490 \mathrm{~nm}$ using a Bio-Rad iMark ${ }^{\mathrm{TM}}$ microplate reader (Bio-Rad Laboratories, Inc.).

Western blotting to measure the protein expression levels of HIF-1 $\alpha$ and VEGF. The cells were directly washed three times with PBS, and lysed in sodium dodecyl sulfate (SDS) sample buffer [50 mM Tris- $\mathrm{HCl}$ (pH 6.8), $1 \%$ SDS, $10 \%$ glycerol, $5 \% \beta$-mercaptoethanol, $0.01 \%$ Bromophenol blue; all reagents purchased from Sigma-Aldrich]. Cell lysates were boiled for $5 \mathrm{~min}$.

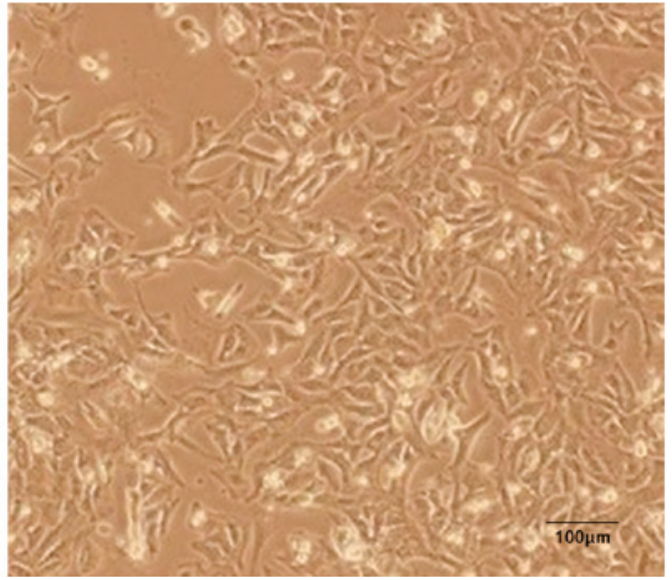

Figure 1. Representative inverted micrograph of a primary culture of myocardial cells from Sprague-Dawley rats.

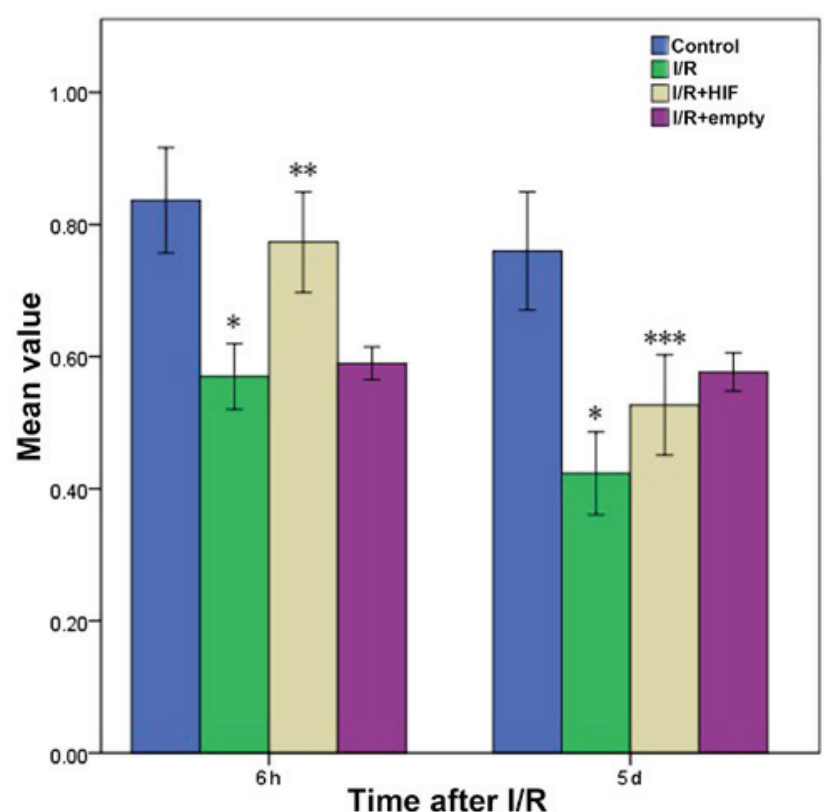

Figure 2. Myocardial cell viability based on the methyl thiazolyl tetrazolium assay at $6 \mathrm{~h}$ or 5 days following treatments simulating I/R. Control cells were subjected to mock treatment; I/R cells were subjected to I/R; HIF-1 $\alpha$ cells were transiently transfected with a plasmid expressing full-length HIF-1 $\alpha$ prior to I/R; and empty cells were transiently transfected with empty expression vector prior to $\mathrm{I} / \mathrm{R}$. The data are shown as the mean \pm standard deviation for three independent experiments. " $\mathrm{P}<0.05$, vs. control; ${ }^{* * *} \mathrm{P}<0.05$, vs. untransfected cells; ${ }^{* * * *} \mathrm{P}<0.05$, vs. $6 \mathrm{~h}$ after $\mathrm{I} / \mathrm{R}$ or mock treatment. I/R, ischemia-reperfusion; HIF-1 $\alpha$, hypoxia-inducible factor- $1 \alpha$.

The supernatants were analyzed by SDS-polyacrylamide gel electrophoresis (10\% gels, run at $85 \mathrm{~V}$ for $45 \mathrm{~min}$, and then at $135 \mathrm{~V}$ for $1 \mathrm{~h}$ ), and subsequently transferred onto nitrocellulose membranes (Bio-Rad Laboratories, Inc.). The filters were blocked with $5 \%$ milk, followed by incubation with a monoclonal antibody against HIF-1 $\alpha$ (1:250), VEGF (1:250) or $\beta$-actin (1:250; all antibodies purchased from ZSGB-BIO, Beijing, China). Binding of the antibodies was detected using the ChemiDoc ${ }^{\mathrm{TM}}$ XRS system (Bio-Rad Laboratories, Inc.).

Flow cytometric analysis to measure apoptosis. Cells were harvested by treatment with trypsin protease (Thermo Fisher Scientific) with subsequent washing with PBS, and then were 
$6 \mathrm{~h}$

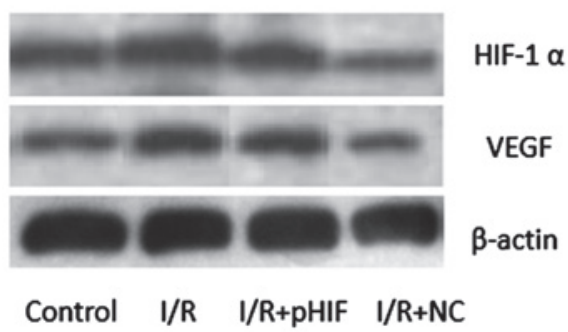

5 day

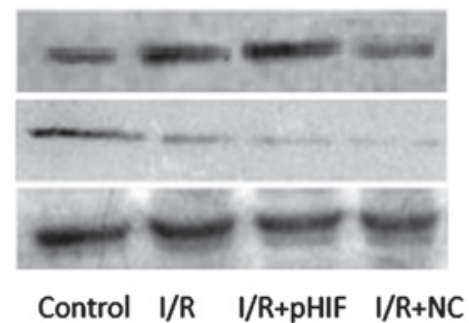

Figure 3. Western blotting of HIF-1 $\alpha$ and the protein VEGF, encoded by a downstream target gene of HIF-1 $\alpha$. Myocardial cells were subjected to treatments simulating I/R (I/R), or they were mock-treated (Control). In parallel, cultures were transiently transfected with empty expression vector (I/R+NC) or an expression plasmid carrying full-length HIF-1 $\alpha$ prior to I/R (I/R+pHIF). Total cell lysates were then prepared at $6 \mathrm{~h}$ and 5 days after I/R or mock treatment, and were subsequently analyzed using appropriate monoclonal antibodies, as detailed in the Materials and methods section. $\beta$-actin was used as a loading control. I/R, ischemia-reperfusion; HIF-1 $\alpha$, hypoxia-inducible factor-1 $\alpha$; VEGF, vascular endothelial growth factor.

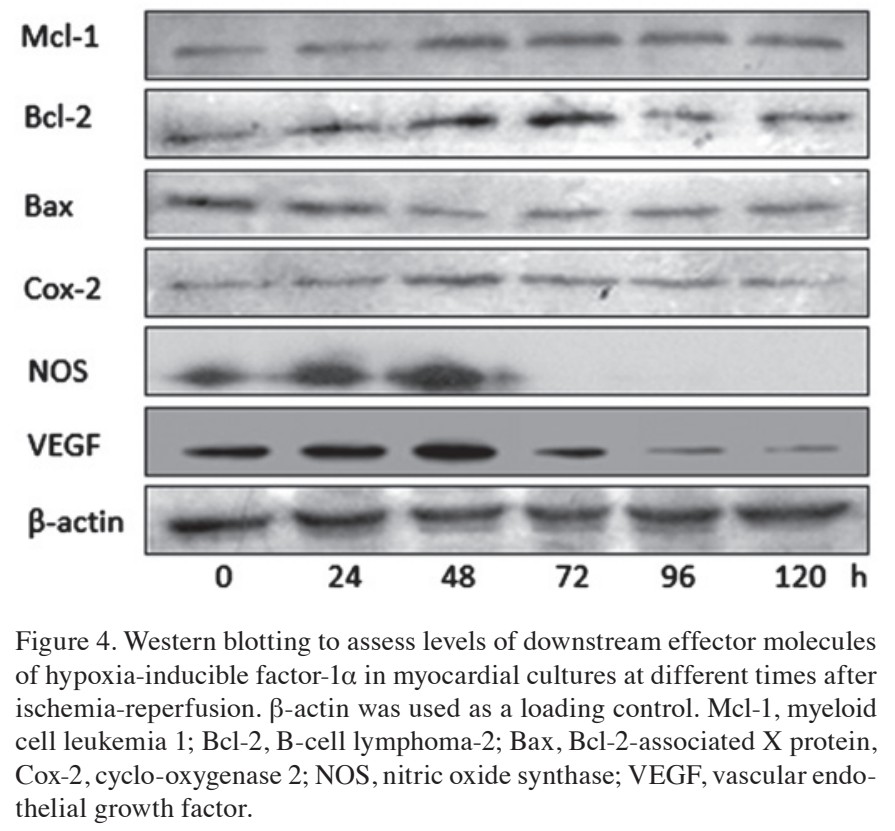

fixed with ethanol (70\%) and maintained at $4^{\circ} \mathrm{C}$ for $30 \mathrm{~min}$. The cells were again pelleted as previously described, the fixation fluid was removed, and the cells were resuspended in $50 \mu \mathrm{l}$ propidium iodide $(0.4 \mathrm{mg} / \mathrm{ml})$. Labeled cells were subsequently analyzed by flow-activated cell sorting using a Becton-Dickson Model 420 Fluorescence-Activated Cell Sorter (BD Biosciences, Bedford, MA, USA).

Statistical analyses. The results for cells harvested at different times following $\mathrm{I} / \mathrm{R}$ with or without prior transfection were analysed by making a comparison of the mean \pm standard deviation. Statistical analyses were performed using SPSS version 19.0 software (IBM SPSS, Armonk, NY, USA). $\mathrm{P}<0.05$ was taken to indicate a statistically significant value.

\section{Results}

Microscopy of the cultures. Inverted microscopy of cultures of the rat myocardial cells revealed the expected morphology of prismatic, or irregular, triangles with ovaloid nuclei centered in the cells (Fig. 1). Analysis of the myocardial cells in DMEM culture dishes showed a pulsation frequency of $100 \pm 10$ beats $/ \mathrm{min}$.
Influence of the expression levels of HIF-1a on myocardial cell viability following $I / R$. At $6 \mathrm{~h}$ following $\mathrm{I} / \mathrm{R}$, cell viability was markedly lower in cells subjected to I/R compared with control cells not subjected to $I / R$, irrespective of whether the cells were untransfected or transfected with empty vector (Fig. 2). Cell viability was markedly higher in cultures transiently transfected with pHIF-1 $\alpha$. At $5 \mathrm{~d}$ following I/R, the viability of these cells was much lower than at $6 \mathrm{~h}$, and was only slightly higher than the viability of untransfected cells and cells transfected with empty vector.

Changes in the expression levels of VEGF and HIF-la following $I / R$. HIF-1 $\alpha$ has been shown to upregulate the expression of VEGF in response to hypoxia. In the culture system utilized in the present study, at $6 \mathrm{~h}$ following I/R, the levels of HIF-1 $\alpha$ and VEGF were higher in cultures treated with $\mathrm{I} / \mathrm{R}$ compared with mock-treated control cells (Fig. 3). Levels were similar at $6 \mathrm{~h}$ for untransfected cells and cells transiently transfected with $\mathrm{pHIF}-1 \alpha$ prior to I/R. At 5 days following I/R, the levels of HIF-1 $\alpha$ remained higher in I/R-treated cells compared with the mock-treated cells. Conversely, the levels of VEGF were markedly lower in I/R-treated cells compared with mock-treated ones.

Western blotting of effector proteins downstream of HIF-1 $\alpha$. The results in Fig. 3 suggested that the hypoxia-induced upregulation of HIF-1 $\alpha$ led to short-term (6 h), but not long-term (5 days), upregulation of VEGF expression. Consequently, it was hypothesized whether the same may also be true for other downstream effector molecules of HIF-1 $\alpha$. Therefore, western blotting was used to assess the levels of B-cell lymphoma-2 (Bcl-2), myeloid cell leukemia 1 (Mcl-1), Bcl-2-associated X protein (Bax), cyclo-oxygenase 2 (Cox-2), nitric oxide synthase (NOS), as well as VEGF at numerous times between 0 and $120 \mathrm{~h}$ following $\mathrm{I} / \mathrm{R}$ in untransfected cells (Fig. 4). These results suggested that, although levels of Bax decreased for much of the time course and potentially increased later, the levels of Mcl-1, Bcl-2, Cox-2, NOS and VEGF peaked at 48-72 h, after which they decreased.

Short-term protection against apoptosis by HIF-1 $\alpha$. The percentage of apoptotic myocardial cells was compared following $I / R$ and mock treatment in cells that were untransfected or transfected prior to I/R with either empty expression vector or expression plasmid encoding full-length 


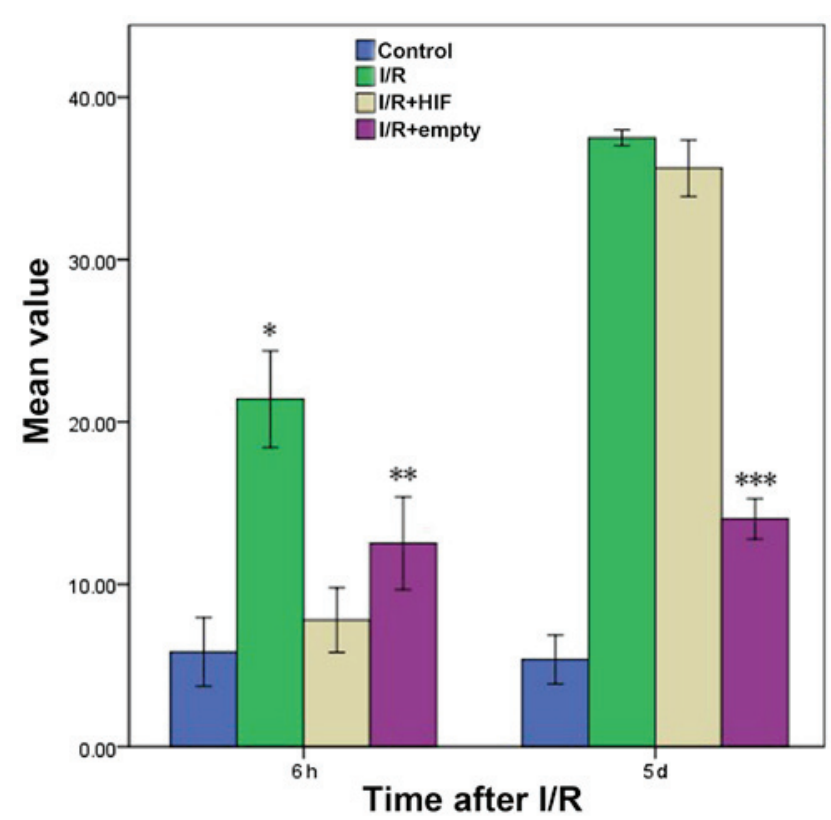

Figure 5. Rate of apoptosis in myocardial cultures following I/R in the presence or absence of HIF-1 $\alpha$ overexpression. Cultures were treated as described in the legend of Fig. 2, and apoptotic cell fractions were determined using fluorescence-activated cell sorting. The bars indicate average values; error bars, the standard deviation ( $\mathrm{n}=3$ for each condition). ${ }^{*} \mathrm{P}<0.05$ vs. control; ${ }^{* *} \mathrm{P}>0.05$ vs. control; ${ }^{* * *} \mathrm{P}<0.05$ vs. $6 \mathrm{~h}$ following I/R or mock treatment. I/R, ischemia-reperfusion; HIF-1 $\alpha$, hypoxia-inducible factor- $1 \alpha$.

HIF-1 $\alpha$ (Fig. 5). Following treatment with I/R, at $6 \mathrm{~h}$ the I/R+HIF group exhibited a similar level of apoptosis with the Control group, which was maintained at a low level compared with the $\mathrm{I} / \mathrm{R}$ and $\mathrm{I} / \mathrm{R}+\mathrm{empty}$ groups. However, at 5 days following I/R, the proportion of apoptotic myocardial cells in the HIF overexpression group had reached a high level, comparable with that of the I/R group.

\section{Discussion}

In the present study, it has been shown that, in a cell culture system that models I/R in animals, the expression of HIF-1 $\alpha$ triggers a short-term upregulation of several downstream effector molecules associated with tissue repair, and the levels of these effectors return to the baseline over the longer term. This occurs even though the levels of HIF-1 $\alpha$ remained relatively high at 5 days following I/R. These findings suggest that the cardioprotective effects of HIF-1 $\alpha$ following hypoxia are temporary.

In contrast with a previous study in mice, which suggested that the levels of HIF-1 $\alpha$ peak soon after hypoxic injury and subsequently rapidly fall back to the baseline (8), in the present study HIF-1 $\alpha$ was detectable at 5 days following I/R-stimulated upregulation of HIF-1 $\alpha$. The difference in these results may reflect different detection systems. The observation that levels of downstream effectors of HIF-1 $\alpha$ decreased faster, and to a greater extent, compared with the levels of HIF-1 $\alpha$ itself suggest that additional factors or pathways are involved in regulating these downstream effectors following $I / R$. It is possible that HIF-1 $\alpha$ initially upregulates these effectors soon after hypoxic injury, and subsequently other factors regulate them in the longer term.
The effectors upregulated in response to $I / R$ in the culture system used in the present study are known to help mitigate the effects of hypoxic injury. Ischemic preconditioning upregulates Cox-2 levels in the heart, helping to protect the tissue against the effects of myocardial infarction (9). In endothelial cells, Cox-2-dependent production of prostacyclin may exert antithrombotic effects (10), and in myocardial cells, adiponectin acts via a Cox2-dependent pathway to mitigate I/R injury (11). $\mathrm{NO}$, which offers one of the most important defenses against myocardial I/R injury (2), is produced by NOS primarily in the cardiovascular system, where it helps to regulate apoptosis. Cox-2 and NO interact to provide cardioprotection, since Cox-2 requires NO for its activity (12). The results of the present study suggest that the protective effects provided by NOS and Cox-2 last for only $\sim 48 \mathrm{~h}$, with their levels approaching the pre-injury baseline following $72 \mathrm{~h}$ (Fig. 4).

These results suggest that HIF-1 $\alpha$ in myocardial cells temporarily increases the expression of the antiapoptotic proteins, Bcl-2 and Mcl-1, while at the same time inhibiting expression of the proapoptotic protein, Bax (Fig. 4). This would be consistent with previous studies, which reported that overexpression of Bcl-2 in cardiac ischemia suppresses cell death and reduces metabolic functions of the mitochondria (10). HIF-1 $\alpha$ appears to exert a similarly short-term effect on VEGF, the levels of which peak at 48-72 h following I/R, and subsequently rapidly return to baseline. VEGF stimulates neovascularization and enhances resistance to ischemia $(1,13)$.

In conclusion, the present study provides evidence that the ability of HIF-1 $\alpha$ and its downstream effectors to attenuate $\mathrm{I} / \mathrm{R}$ injury in myocardial cells is temporary. These findings may have implications for treating such injuries in a variety of clinical contexts.

\section{Acknowledgements}

The present study was supported by the Department of Science and Technology of Sichuan Province, China [grant no. 2011SZ0116] and the Program of Medicine Administration Bureau of Sichuan Province [grant no. 2012-F-024], as well as the Scientific and Technological Project of Sichuan Province [grant no. 6zc1671]. We would like to thank Professor Yi Qu of West China Second University Hospital (Chengdu, China) for her assistance.

\section{References}

1. Eltzschig HK and Collard CD: Vascular ischaemia and reperfusion injury. Br Med Bull 70: 71-86, 2004.

2. Ockaili R, Natarajan R, Salloum F, Fisher BJ, Jones D, Fowler AA III and Kukreja RC: HIF-1 activation attenuates postischemic myocardial injury: Role for heme oxygenase-1 in modulating microvascular chemokine generation. Am J Physiol Heart Circ Physiol 289: H542-H548, 2005.

3. Epstein AC, Gleadle JM, McNeill LA, Hewitson KS, O'Rourke J, Mole DR, Mukherji M, Metzen E, Wilson MI, Dhanda A, et al: C. elegans EGL-9 and mammalian homologs define a family of dioxygenases that regulate HIF by prolyl hydroxylation. Cell 107: 43-54, 2001.

4. Hofer T, Wenger $\mathrm{R}$ and Gassmann M: Oxygen sensing, HIF-1alpha stabilization and potential therapeutic strategies. Pflugers Arch 443: 503-507, 2002.

5. Stroka DM, Burkhardt T, Desbaillets I, Wenger RH, Neil DA, Bauer C, Gassmann M and Candinas D: HIF-1 is expressed in normoxic tissue and displays an organ-specific regulation under systemic hypoxia. FASEB J 15: 2445-2453, 2001. 
6. Lipsky PE, Brooks P, Crofford LJ, DuBois R, Graham D, Simon LS, van de Putte LB and Abramson SB: Unresolved issues in the role of cyclooxygenase-2 in normal physiologic processes and disease. Arch Intern Med 160: 913-920, 2000.

7. Harris AL: Hypoxia - a key regulatory factor in tumour growth. Nat Rev Cancer 2: 38-47, 2002.

8. Bolli R, Shinmura K, Tang XL, Kodani E, Xuan YT, Guo Y and Dawn B: Discovery of a new function of cyclooxygenase (COX)-2: COX-2 is a cardioprotective protein that alleviates ischemia/reperfusion injury and mediates the late phase of preconditioning. Cardiovasc Res 55: 506-519, 2002.

9. Murry CE, Jennings RB and Reimer KA: Preconditioning with ischemia: A delay of lethal cell injury in ischemic myocardium. Circulation 74: 1124-1136, 1986.

10. Adams JM, Difazio LT, Rolandelli RH, Luján JJ, Haskó G, Csóka B, Selmeczy Z and Németh ZH: HIF-1: A key mediator in hypoxia. Acta Physiol Hung 96: 19-28, 2009.
11. Ramírez-Bergeron DL, Runge A, Adelman DM, Gohil M and Simon MC: HIF-dependent hematopoietic factors regulate the development of the embryonic vasculature. Dev Cell 11: 81-92, 2006

12. Date T, Belanger AJ, Mochizuki S, Sullivan JA, Liu LX, Scaria A, Cheng SH, Gregory RJ and Jiang C: Adenovirus-mediated expression of p35 prevents hypoxia/reoxygenation injury by reducing reactive oxygen species and caspase activity. Cardiovasc Res 55: 309-319, 2002.

13. Shibata R, Sato K, Pimentel DR, Takemura Y, Kihara S, Ohashi K, Funahashi T, Ouchi N and Walsh K: Adiponectin protects against myocardial ischemia-reperfusion injury through AMPK- and COX-2-dependent mechanisms. Nat Med 11: 1096-1103, 2005. 